\title{
OSCILLATORY PROPERTIES OF CERTAIN NONLINEAR MATRIX DIFFERENTIAL SYSTEMS OF SECOND ORDER
}

\author{
BY \\ GARRET J. ETGEN
}

Introduction. The use of the polar coordinate transformation in studying the oscillatory properties of certain second order differential equations is well known. W.M. Whyburn [8] introduced this method in order to determine the behavior of solutions of nonlinear second order differential systems of the form

$$
\begin{aligned}
y^{\prime} & =k(x, y, z) \cdot z, & & z^{\prime}=g(x, y, z) \cdot y, \\
y(a) & =0, & z(a)=1 . & a \leqq x \leqq b,
\end{aligned}
$$

J.H. Barrett [2] and W.T. Reid [7] generalized the polar coordinate transformation in order to study the properties of the solutions of the second order linear matrix differential system

$$
\begin{aligned}
Y^{\prime} & =K(x) \cdot Z, \quad Z^{\prime}=G(x) \cdot Y, \\
Y(a) & =0, \quad Z(a)=E(\text { the identity matrix), }
\end{aligned}
$$

where $Y, Z, K(x)$ and $G(x)$ are $n \times n$ matrices and each of $K(x)$ and $G(x)$ is a symmetric matrix of continuous functions on $a \leqq x<\infty$. By a solution of (b) we mean a pair of $n \times n$ matrices $\{Y(x), Z(x)\}$ such that each of the elements of $Y$ and $Z$ is a differentiable function and such that $\{Y, Z\}$ satisfies the initial condition and satisfies the matrix differential system almost everywhere on $a \leqq x<\infty$. The roles of the sine and cosine functions in the polar coordinate transformation are assumed by the solution pair of $n \times n$ matrices $\{S[a, x ; Q], C[a, x ; Q]\}$ of the linear matrix differential system

$$
\begin{aligned}
& Y^{\prime}=Q(x) \cdot Z, \quad Z^{\prime}=-Q(x) \cdot Y, \\
& Y(a)=0, \quad Z(a)=E, \quad a \leqq x<\infty,
\end{aligned}
$$

where $Q(x)$ is a symmetric matrix of continuous functions on $a \leqq x<\infty$.

The purpose of this paper is to investigate the properties of the solution pair $\{S[a, x ; Q], C[a, x ; Q]\}$ of (c), to improve and extend the results of Barrett and

Presented to the Society, January 26, 1965 under the title Oscillatory properties of certain nonlinear differential systems of second order; received by the editors May 10, 1965. 
Reid, and to apply the generalization of the polar coordinate transformation to certain nonlinear matrix differential systems of second order.

The quantities to be used in the paper are confined to the real domain and the notation of matrix algebra and calculus is used throughout. In particular, capital letters are used to denote matrices, small letters denote scalar quantities; the symbol $E$ is used to signify the $n \times n$ identity matrix, while 0 is used indiscriminately for the zero matrix of any dimension. If $A$ is an $n \times n$ matrix, then $\operatorname{tr} A=\sum_{i=1}^{n} a_{i i}$ denotes the trace of $A$ and the transpose of $A$ is denoted $A^{*}$. The symbol $|A|$ signifies the determinant of $A$ and in the event $|A| \neq 0$, the unique inverse exists and is denoted $A^{I}$. The Euclidean norm of $A$ is given by $\|A\|=\left[\sum_{j=1}^{n}\left(\sum_{i=1}^{n} a_{i j}^{2}\right)\right]^{1 / 2}$. If $Y(x)$ is an $n \times n$ matrix each of whose elements is a function, then $Y^{\prime}(x)$ denotes the matrix of derivatives when each of the $n^{2}$ derivatives exists and $\int_{a}^{b} Y(x) d x$ denotes the matrix of integrals when each of the $n^{2}$ integrals exists. The matrix $Y(x)$ is said to be continuous, differentiable, etc., when each of its elements has the indicated property. Finally, since most of the matrices considered in the paper are square with $n$ rows, hereafter, if the term matrix is used without reference to the number of rows or columns, then the matrix is assumed to be square with $n$ rows.

\section{The trigonometric matrices.}

A. Identities. Let $Q(x)$ be a continuous, symmetric, $n \times n$ matrix on $X: 0 \leqq x<\infty$ and consider the second order linear matrix differential system

$$
Y^{\prime}=Q(x) \cdot Z, \quad Z^{\prime}=-Q(x) \cdot Y .
$$

By elementary existence and uniqueness theory (see for example [5] or [9]) there exists a unique solution pair of (1.1) satisfying the initial condition

$$
Y(a)=0, \quad Z(a)=E,
$$

where $a$ is any nonnegative number. This unique solution pair will be denoted by $\{S[a, x ; Q], C[a, x ; Q]\} \equiv\{S[a, x], C[a, x]\}$ when $a>0$, and by $\{S[x ; Q], C[x ; Q]\}$ $\equiv\{S(x), C(x)\}$ when $a=0$.

The theorems of this section will be stated and proved in terms of the pair $\{S[x ; Q], C[x ; Q]\}$ of $(1.1),(1.2)$ with $a=0$. It will be easily seen however, that with trivial modifications in the existing proofs, corresponding theorems hold for any nonnegative number $a$.

Barrett $[2$, Theorem 1.1, p. 511] has shown that the pair $\{S[x ; Q], C[x ; Q]\}$ satisfies the following identities on $X$ :

$$
\begin{gathered}
C^{*}(x) \cdot C(x)+S^{*}(x) \cdot S(x)=E, \quad C^{*}(x) \cdot S(x)=S^{*}(x) \cdot C(x), \\
C(x) \cdot C^{*}(x)+S(x) \cdot S^{*}(x)=E, \quad C(x) \cdot S^{*}(x)=S(x) \cdot C^{*}(x) \\
\|C(x)\|^{2}+\|S(x)\|^{2}=n .
\end{gathered}
$$


The following theorem is a generalization of the trigonometric addition formulas.

THEOREM 1.1. If $b$ is any positive number and $\{S[b, x ; Q], C[b, x ; Q]\}$ is the solution of (1.1), (1.2) with $a=b$, then the following identities hold on $X$ :

$$
\begin{gathered}
S(x)=S(b, x) \cdot C^{*}(b, 0)-C(b, x) \cdot S^{*}(b, 0), \\
C(x)=C(b, x) \cdot C^{*}(b, 0)+S(b, x) \cdot S^{*}(b, 0), \\
S(b, x)=S(x) \cdot C^{*}(b)-C(x) \cdot S^{*}(b), \\
C(b, x)=C(x) \cdot C^{*}(b)+S(x) \cdot S^{*}(b) .
\end{gathered}
$$

Proof. Put $A(x)=S(b, x) \cdot C^{*}(b, 0)-C(b, x) \cdot S^{*}(b, 0)$ and $B(x)=C(b, x)$ - $C^{*}(b, 0)+S(b, x) \cdot S^{*}(b, 0)$. By identity $(1.4), A(0)=0, B(0)=E$, and it is easily verified that $A^{\prime}=Q(x) \cdot B$ and $B^{\prime}=-Q(x) \cdot A$. Thus the pair $\{A(x), B(x)\}$ satisfies (1.1), (1.2) with $a=0$ which implies $\{A(x), B(x)\} \equiv\{S(x), C(x)\}$. Identities (1.8) and (1.9) are established in a similar manner.

B. Oscillatory behavior of the trigonometric matrices. In order to determine the oscillatory properties of the solution pair $\{S[x ; Q], C[x ; Q]\}$, we consider system (1.1) under the further condition that $Q(x)$ is positive definite on $X$. The restriction that $Q(x)$ be positive definite is a natural extension from the case $n=1$; since, if $n=1$, the oscillatory properties of the solution pair $\left\{\sin \int Q\right.$, $\left.\cos \int Q\right\}$ are determined only in case $Q(x)$ is positive on $X$, i.e. only in case $\int_{0}^{x} Q(t) d t$ is an increasing function on $X$.

Definition. A solution $Y(x)$ of $(1.1)$ is said to be oscillatory on $X$ provided for each nonnegative number $b$ there is a number $c, c>b$, such that $|Y(c)|=0$. $Y(x)$ is said to be nonoscillatory if it is not oscillatory.

The following properties of symmetric and symmetric definite matrices are well known and they are used with such frequency in the succeeding work that they will be listed here for reference.

LEMMA A. If $W(x)$ is a continuous, symmetric, positive (negative, nonpositive, nonnegative) definite matrix on $a \leqq x \leqq b$, then $\int_{a}^{x} W(t) d t$ is continuous, symmetric and positive (negative, nonpositive, nonnegative) definite on $a<x \leqq b$.

LEMMA B. If each of $P$ and $Q$ is symmetric with characteristic roots $p_{1} \geqq p_{2} \geqq \cdots \geqq p_{n}$ and $q_{1} \geqq q_{2} \geqq \cdots \geqq q_{n}$ respectively, and if $P-Q$ is nonnegative definite, then $p_{1} \geqq q_{1}, p_{2} \geqq q_{2}, \cdots, p_{n} \geqq q_{n}$.

LEMMA C. If $W(x)$ is a continuous, symmetric matrix on $a \leqq x \leqq b$, then each of the functions $f_{i}(x), i=1,2, \cdots, n$, is real and continuous on $a \leqq x \leqq b$, where for each $x, f_{i}(x)$ is the ith characteristic root of $W(x)$.

The solution pair $\{S[x ; Q], C[x ; Q]\}$ of $(1.1),(1.2)$ has the initial value $\{0, E\}$, 
and $|C[0 ; Q]|=1,|S[0 ; Q]|=0$. Since $C[x ; Q]$ is absolutely continuous, it follows that $|C[x ; Q]|$ is absolutely continuous and thus there is an interval $0 \leqq x<b$ on which $C[x ; Q]$ is nonsingular. To see that there is also an interval over which $S[x ; Q]$ is nonsingular, we prove:

THEOREM 1.2. If $b, b>0$, is the first number such that $C[x ; Q]$ is singular, then $S[x ; Q]$ is nonsingular on $0<x<b$.

Proof. Let $T(x)=S(x) \cdot C^{I}(x)$ on $0 \leqq x<b$. Note that, for $n=1, T(x)$ $=\tan \int_{0}^{x} Q . T(0)=0$; by identity $1.3, T(x)$ is symmetric; and calculating $T^{\prime}(x)$,

$$
T^{\prime}=T \cdot Q \cdot T+Q \text { on } 0 \leqq x<b .
$$

Since $Q(x)$ is positive definite, it follows from Lemma A that $T(x)=\int_{0} T^{\prime}(t) d t$ is positive definite on $0<x<b$. Therefore $S(x)$ must be nonsingular on $0<x<b$.

The following simple example illustrates that $S[x ; Q]$ and $C[x ; Q]$ can be singular simultaneously: In (1.1), (1.2), let $Q(x)$ be the $2 \times 2$ diagonal matrix

$$
Q(x)=\left(\begin{array}{cc}
\pi & 0 \\
0 & \pi / 2
\end{array}\right)
$$

Then

$$
\begin{aligned}
& S[x ; Q]=\left(\begin{array}{cc}
\sin \pi x & 0 \\
0 & \sin (\pi / 2) x
\end{array}\right), \\
& C[x ; Q]=\left(\begin{array}{cc}
\cos \pi x & 0 \\
0 & \cos (\pi / 2) x
\end{array}\right)
\end{aligned}
$$

and $|S[2 n+1 ; Q]|=|C[2 n+1 ; Q]|=0, n=1,2, \cdots$.

This example raises such questions as: $\operatorname{Can} S[x ; Q]$ and /or $C[x ; Q]$ be singular on an interval? If $a \leqq x \leqq b$ is an interval of finite length, can $S[x ; Q]$ and/or $C[x ; Q]$ have infinitely many singularities on $a \leqq x \leqq b$ ? Do the singularities of $S[x ; Q]$ and $C[x ; Q]$ have any separation property?

The following sequence of theorems serves to answer these questions.

THEOREM 1.3. If $a \leqq x \leqq b$ is any interval on which $C[x ; Q]$ is nonsingular, then $S[x ; Q]$ has at most $n$ singularities on $a \leqq x \leqq b$. Similarly, if $S[x ; Q]$ is nonsingular on $c \leqq x \leqq d$, then $C[x ; Q]$ has at most $n$ singularities on $c \leqq x \leqq d$.

Proof. Suppose $C(x)$ is nonsingular on $a \leqq x \leqq b$. Let $T(x)$ be the "tangent" matrix defined on $a \leqq x \leqq b, T(x)=S(x) \cdot C^{I}(x) . T(x)$ is symmetric and $T^{\prime}$ $=T \cdot Q \cdot T+Q$. Let $x_{1}, x_{2}, x_{1}<x_{2}$, be any pair of points on $a \leqq x \leqq b$. Then, by Lemma A, $T\left(x_{2}\right)-T\left(x_{1}\right)=\int_{x_{1}}^{x} T^{\prime}(t) d t=\int_{x_{1}}^{x_{2}}[T(t) \cdot Q(t) \cdot T(t)+Q(t)] d t$ is positive definite. 
For each $x$ on $a \leqq x \leqq b$, let $t_{i}(x), i=1,2, \cdots, n$, denote the $i$ th characteristic root of $T(x)$. By Lemmas B and C, the functions $t_{i}(x)$ are continuous and increasing on $a \leqq x \leqq b$.

Now, the singularities of $S(x)$ coincide with the singularities of $T(x)$ and, since $T(x)$ is symmetric, the singularities of $T(x)$ occur at the zeros of the functions $t_{i}(x)$. As shown above, these functions are increasing on $a \leqq x \leqq b$. Consequently $S(x)$ can have at most $n$ singularities on the interval.

Similarly, if $c \leqq x \leqq d$ is an interval over which $S(x)$ is nonsingular, then consider the "cotangent" matrix $T^{I}(x)=C(x) \cdot S^{I}(x)$ on $c \leqq x \leqq d$. Proceeding as above, it is easily verified that $T^{I}(x)$ has the property that each of its characteristic root functions is decreasing on $c \leqq x \leqq d$. Therefore $C(x)$ can have at most $n$ singularities on $c \leqq x \leqq d$. This completes the proof of the theorem.

This theorem partially answers the question concerning the possibility of $S[x ; Q]$ and /or $C[x ; Q]$ being identically singular on an interval. By the theorem, $S[x ; Q]$ is identically singular on an interval if and only if $C[x ; Q]$ is identically singular on the same interval.

To establish a comparison theorem, let each of $R(x)$ and $Q(x)$ be continuous, symmetric, positive definite, $n \times n$ matrices on $X$ and let the pairs $\{S[x ; R]$, $C[x ; R]\}$ and $\{S[x ; Q], C[x ; Q]\}$ be the solutions of system (1.1), (1.2) with $R(x)$ and $Q(x)$ respectively.

THEOREM 1.4. If $b, b>0$, is the first number such that $C[x ; R]$ is singular, and if $R(x)-Q(x)$ is nonnegative definite on $0 \leqq x<d$ where $d \geqq b$, then $C[x ; Q]$ is nonsingular on $0 \leqq x<b$.

LEMMA 1.4. Let $R(x)$ and $Q(x)$ satisfy the hypothesis of the theorem. If $0 \leqq x<a, a \leqq b$, is the interval over which each of $C[x ; R]$ and $C[x ; Q]$ is nonsingular, and if $T(R)=S[x ; R] \cdot C^{I}[x ; R]$ and $T(Q)=S[x ; Q] \cdot C^{I}[x ; Q]$ on $0 \leqq x<a$, then $T(R)-T(Q)$ is nonnegative definite on $0 \leqq x<a$.

Proof of the lemma. We have seen that each of $T(R)$ and $T(Q)$ is positive definite on $0 \leqq x<a$. Put $A(x)=T(R)-T(Q)$, then

$$
\begin{aligned}
A^{\prime} & =T(R) \cdot R \cdot T(R)+R-T(Q) \cdot Q \cdot T(Q)-Q \\
& =A \cdot B+B^{*} \cdot A+D,
\end{aligned}
$$

where

$$
\begin{aligned}
& B=(1 / 4) \cdot[R+Q] \cdot T(R)+(1 / 4) \cdot[R+Q] \cdot T(Q), \\
& D=(1 / 2) \cdot[T(R) \cdot(R-Q) \cdot T(R)+T(Q) \cdot(R-Q) \cdot T(Q)]+R-Q .
\end{aligned}
$$

$D$ is symmetric and nonnegative definite on $0 \leqq x<a$. According to [6], the homogeneous part of the system $A^{\prime}=A \cdot B+B^{*} \cdot A+D$ has the solution $Y(x)=J^{*}(x) \cdot M \cdot J(x)$, where $M$ is a constant matrix and $J(x)$ is the solution 
$J^{\prime}=J \cdot B, J(0)=E$. By the variation of constants method, the solution of the nonhomogeneous equation is

$$
A(x)=J^{*}(x) \cdot\left[\int_{0}^{x} J^{I *}(t) \cdot D(t) \cdot J^{I}(t) d t\right] \cdot J(x), \quad 0 \leqq x<a .
$$

Since $D(x)$ is nonnegative definite on this interval, it follows, by Lemma A, that $A(x)=T(R)-T(Q)$ is nonnegative definite on $0 \leqq x<a$.

Proof of the theorem. Suppose that the theorem is false, i.e. suppose $C[\mathrm{x} ; Q]$ has a singularity at $x=e, e<b$. Let $a, a \leqq e$, be the first point such that $C[x ; Q]$ is singular and consider $T(R)-T(Q)$ on $0 \leqq x<a . T(Q)=S[x ; Q] \cdot C^{I}[x ; Q]$ and since $C^{\prime}[x ; Q]=-Q(x) \cdot S[x ; Q]$, we have $C^{\prime}[x ; Q]=-Q(x) \cdot T(Q)$ - $C[x ; Q]$. Using Abel's identity, $|C[x ; Q]|=\exp \left\{-\int_{0}^{x} \operatorname{tr}[Q \cdot T(Q)]\right\}$ on $0 \leqq x<a$. Since $|C[a ; Q]|=0$, it follows that $\lim _{x \rightarrow a} \int_{0}^{x} \operatorname{tr}[Q \cdot T(Q)]=\infty$.

On the other hand, since each of $Q(x)$ and $T(Q)$ is positive definite symmetric on $0<x<a, \operatorname{tr}[Q \cdot T(Q)] \leqq[\operatorname{tr} Q] \cdot[\operatorname{tr} T(Q)]$. Also, by hypothesis and the lemma, $[\operatorname{tr} Q] \cdot[\operatorname{tr} T(Q)] \leqq[\operatorname{tr} R] \cdot[\operatorname{tr} T(R)]$. Therefore,

$$
\lim _{x \rightarrow a} \int_{0}^{x} \operatorname{tr}[Q \cdot T(Q)] \leqq \lim _{x \rightarrow a} \int_{0}^{x}[\operatorname{tr} R] \cdot[\operatorname{tr} T(R)]=\int_{0}^{a}[\operatorname{tr} R] \cdot[\operatorname{tr} T(R)]<\infty
$$

since $a<b$. This result contradicts the earlier conclusion that $\lim _{x \rightarrow a} \int_{0}^{x} \operatorname{tr}[Q \cdot T(Q)]=\infty$. Thus the theorem is established.

COROLLARY 1.4.1. If $c, c>0$, is the first number such that $S[x ; R]$ is singular, and if $R(x)-Q(x)$ is nonnegative definite on $0 \leqq x<d$ where $d \geqq c$, then $S[x ; Q]$ is nonsingular on $0<x<c$.

Proof. Obvious modifications in the proof of the theorem yield this corollary. The next corollary is an improvement of the result of Barrett [2, Theorem 3.2 , p. 516].

COROLlaRY 1.4.2. If $b, b>0$, has the property $\int_{0}^{b} \operatorname{tr} Q<\pi / 2$, then $C[x ; Q]$ is nonsingular on $0 \leqq x \leqq b$ and $S[x ; Q]$ is nonsingular on $0<x \leqq b$. If $c, c>0$, has the property $\int_{0}^{c} \operatorname{tr} Q<\pi$, then $S[x ; Q]$ is nonsingular on $0<x \leqq c$ and $C[x ; Q]$ has at most $n$ singularities on $0 \leqq x \leqq c$.

Proof. Let $R(x)=[\operatorname{tr} Q(x)] \cdot E$. Then $S[x ; R]=\left[\sin \int_{0}^{x} \operatorname{tr} Q\right] \cdot E$ and $C[x ; R]=\left[\cos \int_{0}^{x} \operatorname{tr} Q\right] \cdot E . R(x)-Q(x)$ is nonnegative definite symmetric and the theorem applies.

Note that if $\int_{0}^{\infty} \operatorname{tr} Q(x) d x<\pi / 2$, then each of $C[x ; Q]$ and $S[x ; Q]$ is nonsingular on $X$. Moreover, if $\int_{0}^{\infty} \operatorname{tr} Q(x) d x<\pi$, then $S[x ; Q]$ is nonsingular on $0<x<\infty$ and $C[x ; Q]$ has at most $n$ singularities on $X$.

To answer completely the questions raised previously concerning the behavior 
of the singularities of the pair $\{S[x ; Q], C[x ; Q]\}$, we prove the following theorem and corollaries.

THEOREM 1.5. If $a$ is any positive number such that each of $S[a ; Q]$ and $C[a ; Q]$ is nonsingular, and if $b, b>a$, is such that $\int_{a}^{b} \operatorname{tr} Q(t) d t<\pi / 2$, then each of $S[x ; Q]$ and $C[x ; Q]$ has at most $n$ singularities on $a \leqq x \leqq b$.

Proof. Let $a, a>0$, be a number such that each of $S[a ; Q]$ and $C[a ; Q]$ is nonsingular. Such an $a$ exists by Theorem 1.2. Let $b, b>a$, have the property $\int_{a}^{b} \operatorname{tr} Q(x) d x<\pi / 2$.

Consider the pair $\{S[a, x ; Q], C[a, x ; Q]\}$, the solution of (1.1), (1.2) with initial condition at $a>0$. By rephrasing Corollary 1.4.2 in terms of this pair, each of $S[a, x ; Q]$ and $C[a, x ; Q]$ is nonsingular on $a<x \leqq b$.

Put $A(x)=S[a, 0 ; Q] \cdot C^{I}[a, x ; Q] \cdot C[x ; Q]=S(a, 0) \cdot C^{I}(a, x) \cdot C(x)$. It follows from Theorem 1.1 that $S(a, 0)$ is nonsingular and that $A(x)$ is symmetric on $a \leqq x \leqq b$. Calculating $A^{\prime}(x)$, we have

$$
A^{\prime}(x)=S(a, 0) \cdot\left[C^{I}(a, x)\right]^{\prime} \cdot C(x)+S(a, 0) \cdot C^{I}(a, x) \cdot C^{\prime}(x)
$$

which, upon using identities (1.3), (1.4) and Theorem 1.1, reduces to

$$
A^{\prime}(x)=S(a, 0) \cdot C^{l}(a, x) \cdot Q(x) \cdot\left[S(a, 0) \cdot C^{I}(a, x)\right]^{*} .
$$

Thus $A^{\prime}(x)$ is positive definite on $a \leqq x \leqq b$. The fact that $A^{\prime}(x)$ is positive definite implies, as we saw in the proof of Theorem 1.3, that the continuous functions $a_{i}(x)$, where for each $x$ on $a \leqq x \leqq b, a_{i}(x)$ is the $i$ th characteristic root of $A(x)$, are increasing on $a \leqq x \leqq b$. Since the zeros of these functions coincide with the singularities of $C(x)$, it follows that $C(x)$ can have at most $n$ singularities on $a \leqq x \leqq b$.

Letting $B(x)=C[a, 0 ; Q] \cdot C^{I}[a, x ; Q] \cdot S[x ; Q]$ and proceeding as above yields the corresponding conclusion for $S[x ; Q]$.

CoROllaRY 1.5.1. $S[x ; Q]$ and $C[x ; Q]$ cannot be identically singular on an interval. Moreover, the singularities of $S[x ; Q]$ and of $C[x ; Q]$ cannot have a finite limit point.

Proof. Assume that $C(x)$ is identically singular on $a \leqq x \leqq b$. By Theorem $1.3, S(x)$ must also be identically singular on this interval.

Since $Q(x)$ is continuous on $X, \operatorname{tr} Q(x)$ is continuous on $X$ and there exist numbers $c$ and $d$ such that : $c<a$ and each of $C(c)$ and $S(c)$ is nonsingular, $d>a$ and $\int_{c}^{d} \operatorname{tr} Q(t) d t<\pi / 2$. Now, by the theorem, each of $S(x)$ and $C(x)$ has at most $n$ singularities on $c \leqq x \leqq d$, which contradicts the assumption that each of $S(x)$ and $C(x)$ is identically singular on $a \leqq x \leqq b$.

The same contradiction is obtained if it is assumed that the singularities of either $S(x)$ or $C(x)$ have a finite limit point. 
We also have as an immediate consequence of Theorem 1.3 and Corollary 1.5.1, a separation theorem:

Corollary 1.5.2. If $x_{1}$ and $x_{2}$ are consecutive singularities of $S[x ; Q]$, then $C[x ; Q]$ can have at most $n$ singularities on $x_{1} \leqq x \leqq x_{2}$. Similarly, if $y_{1}$ and $y_{2}$ are consecutive singularities of $C[x ; Q]$, then $S[x ; Q]$ can have at most $n$ singularities on $y_{1} \leqq x \leqq y_{2}$.

The concluding theorems of this section establish a necessary and sufficient condition that the "sine" and "cosine" matrices be nonoscillatory on $X$.

THEOREM 1.6. If $\int_{0}^{\infty} \operatorname{tr} Q(t) d t=\infty$ and $b$ is any nonnegative number, then each of $S[x ; Q]$ and $C[x ; Q]$ has infinitely many singularities on $b \leqq x<\infty$.

LEMMA 1.6. If $r$ is a nonnegative number such that $C[x ; Q]$ is nonsingular on $r \leqq x<\infty$ and if $U(x)$ is the matrix defined on $r \leqq x<\infty: U(x)$ $=C(x) \cdot \int_{r}^{x}\left[C^{*}(t) \cdot Q^{I}(t) \cdot C(t)\right]^{I} d t$, then $U(x)$ has the following properties on $r \leqq x<\infty$ :

(i) $U(r)=0$ and $U(x)$ is nonsingular for $x>r$,

(ii) $\|U(x)\|$ is bounded on $r \leqq x<\infty$.

Proof of the lemma. (i) $U(r)=0$ by definition. Since $Q(x)$ is positive definite on $X$ and $C(x)$ is nonsingular on $r \leqq x<\infty$, it follows from Lemma A that $\int_{r}^{x}\left[C^{*} \cdot Q^{I} \cdot C\right]^{I}$ is positive definite on $r<x<\infty$. Thus $U(x)$ is nonsingular on $r<x<\infty$.

(ii) Let $W(x)$ be the matrix defined:

$$
W(x)=-S(x) \cdot \int_{r}^{x}\left[C^{*}(t) \cdot Q^{I}(t) \cdot C(t)\right]^{I} d t+\left[C^{*}(x)\right]^{I}
$$

It is easily verified that the pair $\{U(x), W(x)\}$ is a solution of (1.1) on $r \leqq x<\infty$ satisfying the initial condition $Y(r)=0, Z(r)=\left[C^{*}(r)\right]^{I}$. It is also easily verified that the following identity holds on $r \leqq x<\infty$ :

$$
U^{*}(x) \cdot U(x)+W^{*}(x) \cdot W(x)=\left[C^{*}(r) \cdot C(r)\right]^{I} .
$$

Consequently, upon taking the trace of this identity, we have $\|U(x)\|$ bounded on $r \leqq x<\infty$.

Proof of the theorem. Let $b$ be any nonnegative number and assume that $\int_{0}^{\infty} \operatorname{tr} Q(t) d t=\infty$. Suppose, contrary to the theorem, that $C(x)$ has only a finite number of singularities on $b \leqq x<\infty$. Let $d, d \geqq b$, be the last singularity of $C(x)$ and fix any number $r, r>d$. $C(x)$ is nonsingular on $r \leqq x<\infty$.

Consider the matrix $U(x)$ defined in the lemma and put

$$
V(x)=\int_{r}^{x}\left[C^{*}(t) \cdot Q^{I}(t) \cdot C(t)\right]^{I} d t .
$$


Then $V(x)$ is positive definite symmetric on $r<x<\infty$ and $U(x)=C(x) \cdot V(x)$. Since $C(x)$ is nonsingular on $r \leqq x<\infty, V=C^{I} \cdot U$.

Let $s$ be any number greater than $r$. Since $V$ is positive definite symmetric on $s \leqq x<\infty, V^{I}=U^{I} \cdot C$ has these same properties on $s \leqq x<\infty$. Furthermore, $\left[V^{I}\right]^{\prime}=-V^{I} \cdot V^{\prime} \cdot V^{I}=-U^{I} \cdot Q \cdot\left[U^{I}\right]^{*}$ and therefore $\left[V^{I}\right]^{\prime}$ is negative definite on $s \leqq x<\infty$. Thus, by Lemma $\mathrm{A}$,

$$
\int_{s}^{x}\left[V^{I}\right]^{\prime}=V^{I}(x)-V^{I}(s)=-\int_{s}^{x} U^{I} \cdot Q \cdot\left[U^{I}\right]^{*}
$$

is negative definite on $s<x<\infty$. But, since $V^{I}(x)$ is positive definite on $s \leqq x<\infty$, it follows that $\operatorname{tr} V^{I}(s)-\int_{s}^{x} \operatorname{tr}\left[U^{I} \cdot Q \cdot U^{I_{*}^{*}}\right]>0$. Thus

$$
\int_{s}^{x} \operatorname{tr}\left[U^{I} \cdot Q \cdot U^{I *}\right]<\operatorname{tr} V^{I}(s)<\infty .
$$

Note that since $Q(x)$ is positive definite symmetric,

$$
\operatorname{tr}\left[U^{I} \cdot Q \cdot U^{I *}\right] \geqq\left\|U^{I} \cdot Q \cdot U^{I *}\right\| \geqq\|Q\| \cdot\|U\|^{-2} \geqq(1 / n) \cdot\|U\|^{-2} \cdot \operatorname{tr} Q .
$$

Therefore $\int_{s}^{\infty}[\operatorname{tr} Q] \cdot\|U\|^{-2}<\infty$. But, by the lemma, $\|U(x)\|$ is bounded. Consequently $\int_{s}^{\infty} \operatorname{tr} Q(t) d t<\infty$, contradicting the hypothesis of the theorem. Thus $C(x)$ must have infinitely many singularities on $b \leqq x<\infty$.

In exactly the same manner, it may be shown that $S(x)$ has infinitely many singularities on $b \leqq x<\infty$. Thus the theorem is established.

THEOREM 1.7. A necessary and sufficient condition that each of $S[x ; Q]$ and $C[x ; Q]$ has at most a finite number of singularities on $X$, i.e. that each of $S[x ; Q]$ and $C[x ; Q]$ be nonoscillatory, on $X$, is: $\int_{0}^{\infty} \operatorname{tr} Q(t) d t<\infty$.

Proof. The necessity of the condition is a consequence of the preceding theorem. Thus it remains to show that if $\int_{0}^{\infty} \operatorname{tr} Q=k<\infty$, then each of $S(x)$ and $C(x)$ has at most a finite number of singularities on $X$.

Suppose $\int_{0}^{\infty} \operatorname{tr} Q=k<\infty$. Since $Q(x)$ is positive definite and continuous on $X, \operatorname{tr} Q(x)$ is positive and continuous on $X$ implying that $\int_{0}^{x} \operatorname{tr} Q$ is an increasing function on $X$. Since we have assumed that the improper integral converges, there exists a nonnegative number $r$ such that $\int_{r}^{\infty} \operatorname{tr} Q<\pi / 2$. As a consequence of the preceding theorems, $r$ can be chosen such that each of $S(r)$ and $C(r)$ is nonsingular. Now, by Theorem 1.5, each of $S(x)$ and $C(x)$ has at most $n$ singularities on $r \leqq x<\infty$; and, by Corollary 1.5.1, each of $S(x)$ and $C(x)$ has at most a finite number of singularities on $0 \leqq x \leqq r$. Therefore each of $S(x)$ and $C(x)$ has at most a finite number of singularities on $X$ and the theorem is proved.

It has been the purpose of this section to investigate the properties of solutions of the system (1.1), (1.2), and, in particular, to determine the oscillatory behavior of the solution pairs $\{S[a, x ; Q], C[a, x ; Q]\}$. With the exception of the fact that 
$S[a, x ; Q]$ and $C[a, x ; Q]$ can be singular simultaneously, it has been shown that the "sine" and "cosine" matrices have oscillatory properties quite similar to those of the sine and cosine functions. Moreover, each of the theorems presented in this section specialize in the case $n=1$ to a corresponding theorem for the sine and cosine functions.

2. A nonlinear matrix system. Let $H$ be a subset of real Euclidean $n^{2}$ space and let $\Phi_{H}$ be the collection of functions to which $\phi$ belongs only in case $\phi$ is a continuous, real-valued function from $H$.

Definition. If $Y$ is an $n \times n$ matrix and $\phi$ is a member of $\Phi_{H}$, then $\phi(Y)=\phi\left(y_{11}, y_{12}, \cdots, y_{1 n}, y_{21}, \cdots, y_{n n}\right)$.

Let $\phi_{i j}, \sigma_{i j}, \tau_{i j}, \delta_{i j},(i, j=1,2, \cdots, n)$, be members of $\Phi_{H}$ and consider the following system of $2 n^{2}$ nonlinear differential equations:

$$
\begin{aligned}
& y_{i j}^{\prime}=\sum_{h=1}^{n} k_{i h}\left[x, \phi_{i h}(Y), \sigma_{i h}(Z)\right] \cdot z_{h j}, \\
& z_{i j}^{\prime}=\sum_{h=1}^{n} g_{i h}\left[x, \tau_{i h}(Y), \delta_{i h}(Z)\right] \cdot y_{h j},
\end{aligned}
$$

on $X: 0 \leqq x<\infty$, where the following conditions are imposed:

(i) The real-valued functions $k_{i j}[x, u, v]$ and $g_{i j}[x, u, v]$ are continuous in $(x, u, v) \mathrm{f} ;$ all $x$ on $X$ and all real values of $u$ and $v$.

(ii) T. . re exists a continuous function $m(x)$ such that for all $x$ on $X$ and all real values of $u$ and $v$,

$$
\begin{aligned}
& \left|k_{i j}[x, u, v]\right| \leqq m(x), \\
& \left|g_{i j}[x, u, v]\right| \leqq m(x) .
\end{aligned} \quad(i, j=1, \cdots, n)
$$

(iii) The real-valued functions $k_{i j}[x, u, v]$ and $g_{i j}[x, u, v], i, j=1, \cdots, n$, satisfy a Lipschitz condition with respect to $u$ and $v$.

It is well known (see for example [9]) that when appropriate initial conditions are specified, system (2.1) has a unique solution. Note that condition (iii) may be replaced by any other condition which will insure a unique solution of system (2.1) with conditions (i) and (ii).

System (2.1) will be represented hereafter in the matrix form

$$
\begin{aligned}
& Y^{\prime}=K[x ; Y ; Z] \cdot Z, \quad \text { on } X . \\
& Z^{\prime}=G[x ; Y ; Z] \cdot Y
\end{aligned}
$$

The following theorem is the generalization to system (2.2) of the results of Barrett [2] and Reid [7].

THEOREM 2.1. If, in system (2.2), the matrices $K[x ; Y ; Z]$ and $G[x ; Y ; Z]$ 
are symmetric and $\{Y(x), Z(x)\}$ is the solution pair of (2.2) satisfying the initial condition

$$
Y(0)=0, \quad Z(0)=A, \quad A \text { nonsingular },
$$

then there exists a continuous, symmetric matrix $Q(x)$ and a nonsingular, continuously differentiable matrix $R(x)$ such that

$$
\begin{aligned}
& Y(x)=S^{*}[x ; Q] \cdot R(x), \\
& Z(x)=C^{*}[x ; Q] \cdot R(x) .
\end{aligned}
$$

Furthermore, $R(x)$ satisfies the matrix differential system

$$
\begin{gathered}
R^{\prime}(x)=\left\{S[x ; Q] \cdot K \cdot C^{*}[x ; Q]+C[x ; Q] \cdot G \cdot S^{*}[x ; Q]\right\} \cdot R(x) \\
R(0)=A,
\end{gathered}
$$

and $Q(x)$ is the matrix

$$
Q(x)=C[x ; Q] \cdot K \cdot C^{*}[x ; Q]-S[x ; Q] \cdot G \cdot S^{*}[x ; Q] .
$$

Proof. Proceeding as in [2], let $A$ be a given nonsingular matrix and let $\{Y(x), Z(x)\}$ be the solution of (2.2), (2.3). Also, let $Q_{0}(x)$ be any symmetric, continuous matrix on $X$ with $\left\{S_{0}\left[x ; Q_{0}\right], C_{0}\left[x ; Q_{0}\right]\right\}=\left\{S_{0}, C_{0}\right\}$ the corresponding solution of (1.1), (1.2) with $a=0$.

Define a sequence $\left\{Q_{p}(x)\right\}_{1}^{\infty}$ of symmetric, continuous matrices as follows: for each $Q_{p}(x)$, let $\left\{S\left[x ; Q_{p}\right], C\left[x ; Q_{p}\right]\right\}=\left\{S_{p}, C_{p}\right\}, p=1,2, \cdots$, be the solution of (1.1), (1.2) with $a=0$, and let

$$
Q_{p+1}=C_{p} \cdot K \cdot C_{p}^{*}-S_{p} \cdot G \cdot S_{p}^{*} \quad(p=0,1,2, \cdots) .
$$

Now

$$
\begin{aligned}
Q_{p+1}-Q_{p}= & (1 / 2) \cdot\left(C_{p}-C_{p-1}\right) \cdot K \cdot\left(C_{p}^{*}+C_{p-1}^{*}\right) \\
& +(1 / 2) \cdot\left(C_{p}+C_{p-1}\right) \cdot K \cdot\left(C_{p}^{*}-C_{p-1}^{*}\right) \\
& +(1 / 2) \cdot\left(S_{p-1}-S_{p}\right) \cdot G \cdot\left(S_{p-1}^{*}+S_{p}^{*}\right) \\
& +(1 / 2) \cdot\left(S_{p-1}+S_{p}\right) \cdot G \cdot\left(S_{p-1}^{*}-S_{p}^{*}\right) .
\end{aligned}
$$

Barrett [2, Lemma 2.1, p. 513] established that the following inequalities hold on $X$ :

and

$$
\left\|S_{i}-S_{j}\right\| \leqq 2 n \cdot \int_{0}^{x}\left\|Q_{i}-Q_{j}\right\|
$$

$$
\left\|C_{i}-C_{j}\right\| \leqq 2 n \cdot \int_{0}^{x}\left\|Q_{i}-Q_{j}\right\|
$$


Using this result and Identity 1.5 , we find that there is a constant $m$ such that

$$
\left\|Q_{p+1}-Q_{p}\right\| \leqq m \cdot(\|K\|+\|G\|) \cdot \int_{0}^{x}\left\|Q_{p}-Q_{p-1}\right\| .
$$

Now, by the standard techniques of successive approximation, it is easy to show that the sequence of matrices $\left\{Q_{p}(x)\right\}_{0}^{\infty}$ converges uniformly with respect to the norm on each closed subinterval of $X$. Let $Q(x)$ be the "limit" matrix. $Q(x)$ is continuous and symmetric on $X$ since each of the matrices $Q_{p}(x)$ has these properties and the convergence is uniform on closed subintervals.

Let $\{S[x ; Q], C[x ; Q]\}=\{S(x), C(x)\}$ be the solution of (1.1), (1.2) with $a=0$. It follows from the lemma quoted above that the sequences $\left\{S_{p}(x)\right\}_{0}^{\infty}$ and $\left\{C_{p}(x)\right\}_{0}^{\infty}$ have as their "limits" the matrices $S(x)$ and $C(x)$ respectively. Thus

$$
Q(x)=C(x) \cdot K \cdot C^{*}(x)-S(x) \cdot G \cdot S^{*}(x) .
$$

Define $R(x)$ to be the solution of the system

$$
U^{\prime}=\left[S(x) \cdot K \cdot C^{*}(x)+C(x) \cdot G \cdot S^{*}(x)\right] \cdot U, \quad U(0)=A .
$$

From Abel's Identity, we can conclude that the matrix $R(x)$ is nonsingular on $X$.

To show that (2.4) holds, let $V(x)$ and $W(x)$ be the matrices $S^{*}(x) \cdot R(x)$ and $C^{*}(x) \cdot R(x)$ respectively. It is now a simple matter to verify that the pair $\{V(x), W(x)\}$ satisfies $(2.2)$ and since $V(0)=0$ and $W(0)=A$, it follows that $V(x)=Y(x)$ and $W(x)=Z(x)$ on $X$. The proof of the theorem is now complete.

It is of interest to note that the theorem also holds for the system (2.2) together with the initial condition

$$
Y(0)=0, \quad Z(0)=B, \quad B \text { singular } .
$$

However, in this case, the continuously differentiable matrix $R(x)$, which would be defined as the solution of $(2.5), R(0)=B$, is identically singular on $X$. Consequently, if the pair $\{Y(x), Z(x)\}$ is the solution of (2.2), (2.8), then each of $Y(x)$ and $Z(x)$ is identically singular on $X$.

THEOREM 2.2. Let $m(x)$ be the continuous bound on the elements $k_{i j}, g_{i j}, i, j=1,2, \cdots, n$, of the matrices $K$ and $G$ in system (2.2). If $\{Y(x), Z(x)\}$ is the solution pair of (2.2), (2.3), then

$$
\|Y(x)\|+\|Z(x)\| \leqq\|A\| \cdot \exp \left[\int_{0}^{x} n^{2} \cdot m(t) d t\right] \text { on } X .
$$

Proof. Let $\left.D_{L} f x\right)$ and $D_{R} f(x)$ denote the left and right hand derivatives of $f(x)$ respectively. If $U(x)$ is a differentiable matrix on $X$, then it is well known (see for example [3]) that each of $D_{L}\|U(x)\|$ and $D_{R}\|U(x)\|$ exists for each $x$ on $X$ and, moreover, $\left|D_{L}\|U(x)\|\right| \leqq\left\|U^{\prime}(x)\right\|$ and $\left|D_{R}\|U(x)\|\right| \leqq\left\|U^{\prime}(x)\right\|$ on $X$. 
Now

$$
\begin{aligned}
& \left\|Y^{\prime}(x)\right\| \leqq\|K[x ; Y ; Z]\| \cdot\|Z(x)\| \leqq n^{2} \cdot m(x) \cdot\|Z(x)\|, \\
& \left\|Z^{\prime}(x)\right\| \leqq\|G[x ; Y ; Z]\| \cdot\|Y(x)\| \leqq n^{2} \cdot m(x) \cdot\|Y(x)\| .
\end{aligned}
$$

Adding these inequalities and applying the above result, we have

$$
D_{R}\{\|Y(x)\|+\|Z(x)\|\} \leqq n^{2} \cdot m(x)\{\|Y(x)\|+\|Z(x)\|\} .
$$

It now follows that

$$
\|Y(x)\|+\|Z(x)\| \leqq\|A\| \cdot \exp \left[\int_{0}^{x} n^{2} \cdot m(t) d t\right] \quad \text { on } X .
$$

For the remainder of this section, we shall consider those systems of the form (2.2) in which the following conditions are satisfied: (i), (ii), (iii) stated at the beginning of the section, and

(iv) There exists a positive number $r$ such that the matrices $K[x ; Y ; Z]$ and $-G[x ; Y ; Z]$ are symmetric and positive definite for all $x$ on $X$ and all absolutely continuous matrices $Y(x), Z(x)$ such that

$$
\|Y(x)\|+\|Z(x)\| \leqq r \cdot \exp \left[\int_{0}^{x} n^{2} \cdot m(t) d t\right] \text { on } X .
$$

In particular then, we will be considering those solutions of (2.2), (2.3) which have the property $\|A\| \leqq r$.

THEOREM 2.3. If $\{Y(x), Z(x)\}$ is a solution pair of system (2.2), (2.3), then the matrix $Q(x)$,

$$
Q(x)=C[x ; Q] \cdot K[x ; Y ; Z] \cdot C^{*}[x ; Q]-S[x ; Q] \cdot G[x ; Y ; Z] \cdot S^{*}[x ; Q]
$$

has the following properties on $X$ :

(i) $Q(x)$ is positive definite,

(ii) $\operatorname{tr} Q(x)<2 \max [\operatorname{tr} K, \operatorname{tr}(-G)]$ for each $x$.

Proof. (i) Let $\phi$ be any nonzero constant vector. Since each of $K$ and $-G$ is positive definite and

$$
\begin{aligned}
\phi \cdot C \cdot C^{*} \cdot \phi^{*}+\phi \cdot S \cdot S^{*} \cdot \phi^{*} & =(\phi \cdot C) \cdot(\phi \cdot C)^{*}+(\phi \cdot S) \cdot(\phi \cdot S)^{*} \\
& =\phi \cdot \phi^{*}>0,
\end{aligned}
$$

we have

$$
\phi \cdot Q \cdot \phi^{*}=(\phi \cdot C) \cdot K \cdot(\phi \cdot C)^{*}-(\phi \cdot S) \cdot G \cdot(\phi \cdot S)^{*}>0 .
$$

Thus $Q(x)$ is positive definite on $X$.

LEMMA 2.3. If $A$ is any positive definite symmetric matrix, then

$$
\operatorname{tr} A=\operatorname{tr}\left(C[x ; Q] \cdot A \cdot C^{*}[x ; Q]\right)+\operatorname{tr}\left(S[x ; Q] \cdot A \cdot S^{*}[x ; Q]\right) .
$$


Proof of the lemma. Since $A$ is positive definite symmetric, there exists a matrix $N$ such that $A=N \cdot N^{*}$ and $\operatorname{tr} A=\operatorname{tr} N \cdot N^{*}=\operatorname{tr} N^{*} \cdot N$. Now, from identity (1.3).

$$
N^{*} \cdot N=N^{*} \cdot C^{*} \cdot C \cdot N+N^{*} \cdot S^{*} \cdot S \cdot N
$$

Taking the trace of this equation yields

$$
\begin{aligned}
\operatorname{tr} A=\operatorname{tr} N^{*} \cdot N & =\operatorname{tr}(C \cdot N)^{*} \cdot(C \cdot N)+\operatorname{tr}(S \cdot N)^{*} \cdot(S \cdot N) \\
& =\operatorname{tr}(C \cdot N) \cdot(C \cdot N)^{*}+\operatorname{tr}(S \cdot N) \cdot(S \cdot N)^{*} \\
& =\operatorname{tr}\left(C \cdot N \cdot N^{*} \cdot C^{*}\right)+\operatorname{tr}\left(S \cdot N \cdot N^{*} \cdot S^{*}\right) \\
& =\operatorname{tr} C \cdot A \cdot C^{*}+\operatorname{tr} S \cdot A \cdot S^{*} .
\end{aligned}
$$

Proof of the theorem. (ii) Let $P(x)$ be the matrix

$$
P(x)=S[x ; Q] \cdot K[x ; Y ; Z] \cdot S^{*}[x ; Q]-C[x ; Q] \cdot G[x ; Y ; Z] \cdot C^{*}[x ; Q] .
$$

By part (i) of this theorem, $P(x)$ is symmetric and positive definite on $X$.

Now, by the lemma,

$$
\begin{aligned}
\operatorname{tr} Q+\operatorname{tr} P & =\operatorname{tr}\left[C \cdot K \cdot C^{*}-S \cdot G \cdot S^{*}\right]+\operatorname{tr}\left[S \cdot K \cdot S^{*}-C \cdot G \cdot C^{*}\right] \\
& =\operatorname{tr} K+\operatorname{tr}(-G) .
\end{aligned}
$$

Since $P(x)$ is positive definite on $X, \operatorname{tr} P>0$ on $X$ and consequently

$$
\operatorname{tr} Q<\operatorname{tr} K+\operatorname{tr}(-G)<2 \cdot \max [\operatorname{tr} K, \operatorname{tr}(-G)]
$$

for each $x$ on $X$. This completes the proof of the theorem.

If $\{Y(x), Z(x)\}$ is a solution pair of (2.2), (2.3) such that $\|Z(0)\|=\|A\| \leqq r$, then the pair $\{Y(x), Z(x)\}$ has the following oscillatory behavior on $X$ :

THEOREM 2.4. If $b$ is a positive number having the property

$$
\int_{0}^{b} \max \{\operatorname{tr} K[x ; Y ; Z], \operatorname{tr}(-G[x ; Y ; Z])\}<\pi / 4,
$$

then $Z(x)$ is nonsingular on $0 \leqq x \leqq b$ and $Y(x)$ is nonsingular on $0<x \leqq b$. If $c$ is a positive number having the property

$$
\int_{0}^{c} \max \{\operatorname{tr} K[x ; Y ; Z], \operatorname{tr}(-G[x ; Y ; Z])\}<\pi / 2,
$$

then $Y(x)$ is nonsingular on $0<x \leqq c$ and $Z(x)$ has at most $n$ singularities on $0 \leqq x \leqq c$.

Proof, By Theorems 2.3, 2.1 and Corollary 1.4.2. 
THEOREM 2.5. If $a$ is any positive number such that each of $Y(a)$ and $Z(a)$ is nonsingular, and if $b, b>a$, has the property

$$
\int_{a}^{b} \max \operatorname{tr}\{K[x ; Y ; Z], \operatorname{tr}(-G[x ; Y ; Z])\}<\pi / 4
$$

then each of $Y(x)$ and $Z(x)$ has at most $n$ singularities on $a \leqq x \leqq b$.

Proof. By Theorem 2.3, 2.1 and 1.5.

THEOREM 2.6. If $a$ is any nonnegative number and $b$ is any number greater than $a$, then each of $Y(x)$ and $Z(x)$ has at most a finite number of singularities on $a \leqq x \leqq b$.

Proof. By Theorems 2.3, 2.1 and Corollary 1.5.1.

Theorem 2.7 (SeParation Theorem). If $x_{1}$ and $x_{2}$ are consecutive singularities of $Y(x)$, then $Z(x)$ has at most $n$ singularities on $x_{1} \leqq x \leqq x_{2}$. Similarly, if $y_{1}$ and $y_{2}$ are consecutive singularities of $Z(x)$, then $Y(x)$ has at most $n$ singularities on $y_{1} \leqq x \leqq y_{2}$.

Proof. By Theorem 2.1 and Corollary 1.5.2.

We conclude this section with the following sufficient condition that the pair $\{Y(x), Z(x)\}$ be nonoscillatory on $X$.

THEOREM 2.8. If each of $\int_{0}^{\infty} \operatorname{tr} K[x ; Y ; Z]$ and $\int_{0}^{\infty} \operatorname{tr}(-G[x ; Y ; Z]$ is finite, then each of $Y(x)$ and $Z(x)$ has at most a finite number of singularities on $X$.

Proof. By Theorems 2.3, 2.1 and 1.7.

In conclusion, it is worthwhile noting that the linear system

$$
\begin{aligned}
Y^{\prime} & =K(x) \cdot Z, \\
Z^{\prime} & =G(x) \cdot Y, \\
Y(0) & =0, \quad Z(0)=A, \quad A \text { nonsingular, }
\end{aligned}
$$

where each of $K(x)$ and $-G(x)$ is continuous, symmetric and positive definite on $X$, occurs as a special case of the foregoing treatment. Hence Theorems 2.1 through 2.8 hold for all solutions $\{Y(x), Z(x)\}$ of (2.10), (2.11).

3. The nonlinear system depending upon a parameter. Before considering the nonlinear system, we first establish an additional property of the trigonometric matrices of $\$ 1$.

Let $Q(x, \psi)$ be an $n \times n$ symmetric matrix which is continuous on

$$
X: 0 \leqq x<\infty, \quad L: d_{1}<\psi<d_{2} .
$$

As in $\S 1$, consider the second order matrix differential system 


$$
\begin{gathered}
d Y / d x=Q(x, \psi) \cdot Z, \quad d Z / d x=-Q(x, \psi) \cdot Y \quad \text { on } X L, \\
Y(a, \psi) \equiv 0, \quad Z(a, \psi) \equiv E, \quad a \geqq 0,
\end{gathered}
$$

where the following conditions are imposed on the matrix $Q(x, \psi)$ :

(i) $Q(x, \psi)$ is continuous in $(x, \psi)$ on $X L$,

(ii) $Q(x, \psi)$ is symmetric and positive definite for all values of $x$ and $\psi$ on $X L$,

(iii) there exists a positive number $m(Q)$ such that if $q_{i}(x, \psi), i=1,2, \cdots, n$, is the $i$ th characteristic root of $Q(x, \psi)$ for each $(x, \psi)$ and $\lambda(x)=\min q_{i}(x, \psi), 1 \leqq i \leqq n, x$ on $X$, for all $\psi$ on $L$, then for each $x$ on $X$,

$$
[\operatorname{tr} Q(x, \psi)] / \lambda(x) \leqq m(Q)
$$

for all $\psi$ on $L$.

Observe that condition (iii) imposes a type of uniformity in the behavior of the characteristic root functions of $Q(x, \psi)$. This condition can be stated equivalently as follows: if $\mu(x)=\max q_{i}(x, \psi), 1 \leqq i \leqq n, x$ on $X$, for all $\psi$ on $L$, then there exists a positive number $m^{\prime}(Q)$ such that $\mu(x) / \lambda(x) \leqq m^{\prime}(Q)$ for all $x$ on $X$ and all $\psi$ on $L$.

The solution of system (3.1), (3.2) will be denoted by $\{S[a, x ; Q ; \psi]$, $C[a, x ; Q ; \psi]\} \equiv\{S[a, x, \psi], C[a, x, \psi]\}$ when $a>0$ and by $\{S[x ; Q ; \psi]$, $C[x ; Q ; \psi]\} \equiv\{S(x, \psi), C(x, \psi)\}$ when $a=0$. The properties of the "sine" and "cosine" matrices developed in $\$ 1$ obviously hold for the solution pairs of (3.1), (3.2) for each fixed $\psi$ on $L$.

THEOREM 3.1. To each $Q(x, \psi)$ satisfying conditions (i), (ii) and (iii) there corresponds a positive number $q$ such that if $\psi$ is any fixed number on $L, a$ is any nonnegative number and $b, b>a$, has the property $\int_{a}^{b} \operatorname{tr} Q(x, \psi) d x \geqq q$, then each of $S[x ; Q ; \psi]$ and $C[x ; Q ; \psi]$ has at least one singularity on $a \leqq x \leqq b$.

LEMMA 3.1.1. If $Q(x)$ is any symmetric positive definite matrix on $X$ and $a$ is any nonnegative number with $\{S[a, x ; Q], C[a, x ; Q]\}$ the solution of (1.1), (1.2), and if $b, b>a$, is the next singularity of $S[a, x ; Q]$, then each of $S[x ; Q]$ and $C[x ; Q]$ has at least one singularity on $a \leqq x \leqq b$.

Proof of the lemma. If $a=0$, then $\{S[a, x ; Q], C[a, x ; Q]\} \equiv\{S[x ; Q]$, $C[x ; Q]\}$ and the lemma follows from Theorem 1.2.

Suppose $a>0$. If each of $S(a)$ and $C(a)$ is singular, then we are done. Suppose $S(a)$ is nonsingular and assume that $S(x)$ is nonsingular on $a<x \leqq b$. Put $A(x)=S^{\mathrm{I}}(x) \cdot S[a, x] \cdot S^{I *}(a)$ on $a \leqq x \leqq b$. By the "addition" identity (1.8), $A(x)=S^{I}(a) \cdot C(a)-S^{I}(x) \cdot C(x)$. Thus $A(x)$ is symmetric on this interval. Also $A(a)=0$ and $A^{\prime}(x)=S^{I}(x) \cdot Q(x) \cdot S^{I *}(x)$ is positive definite on $a \leqq x \leqq b$. Consequently, by Lemma $\mathrm{A}, \S 1, A(x)$ is positive definite on $a<x \leqq b$ contradicting the fact that $S[a, b]$ is singular. Thus $S(x)$ must have at least one singularity on $a \leqq x \leqq b$. 
Similarly, if $C(a)$ is nonsingular and we assume that $C(x)$ is nonsingular on $a<x \leqq b$, then, letting $B(x)=C^{I}(x) \cdot S[a, x] \cdot C^{I *}(a)$ and proceeding as above, we obtain the same contradiction. Thus $C(x)$ must have at least one singularity on $a \leqq x \leqq b$ and the lemma is proved.

LEMMA 3.1.2. To each $Q(x, \psi)$ satisfying conditions (i), (ii) and (iii) there corresponds a positive number $q$ such that if $\psi$ is any fixed number on $L, a$ is any nonnegative number and $b, b>a$, has the property $\int_{a}^{b} \operatorname{tr} Q(x, \psi) d x \geqq q$, then each of $S[a, x ; Q ; \psi]$ and $C[a, x ; Q ; \psi]$ has at least one singularity on $a<x \leqq b$.

Proof of the lemma. Put $P(x)=\lambda(x) \cdot E$, where $\lambda(x)$ is the continuous function defined in condition (iii). $P(x)$ is symmetric and positive definite on $X$ and the solution pair of (1.1), (1.2) with $P(x)$ in place of $Q(x)$ is easily seen to be $\{S[a, x ; P], \quad C[a, x ; P]\}=\left\{\left[\sin \int_{a}^{x} \lambda(t) d t\right] \cdot E,\left[\cos \int_{a}^{x} \lambda(t) d t\right] \cdot E\right\}$. Thus if $b$, $b>a$, has the property $\int_{a}^{b} \lambda(t) d t \geqq \pi$, then each of $S[a, x ; P]$ and $C[a, x ; P]$ has at least one singularity on $a<x \leqq b$.

Suppose $\psi$ is fixed and $b, b>a$, has the property

$$
\int_{a}^{b} \operatorname{tr} Q(x, \psi) d x \geqq m(Q) \cdot \pi .
$$

Then

$$
\int_{a}^{b} \lambda(x) d x \geqq[1 / m(Q)] \cdot \int_{a}^{b} \operatorname{tr} Q(x, \psi) d x \geqq \pi .
$$

Consequently, each of $S[a, x ; P]$ and $C[a, x ; P]$ has at least one singularity on $a<x \leqq b$. Now, since $Q(x, \psi)-P(x)$ is nonnegative definite, it follows from Theorem 1.4 that each of $S[a, x ; Q ; \psi]$ and $C[a, x ; Q ; \psi]$ has at least one singularity on $a<x \leqq b$.

Proof of the theorem. Fix any number $\psi$ on $L$ and let $a$ be any nonnegative number. Put $q=m(Q) \cdot \pi$ and suppose $b, b>a$, has the property $\int_{a}^{b} \operatorname{tr} Q(x, \psi) d x$ $\geqq q$. By Lemma 3.1.2, $S[a, x ; Q ; \psi]$ has at least one singularity on $a<x \leqq b$. Thus, by Lemma 3.1.1, each of $S[x ; Q ; \psi]$ and $C[x ; Q ; \psi]$ has at least one singularity on the interval $a \leqq x \leqq b$. This completes the proof of the theorem.

Let $\Phi_{H}$ be the collection of functions defined in $\S 2$. Let $\phi_{i j}, \sigma_{i j}, \tau_{i j}, \delta_{i j}, i, j$ $=1,2, \cdots, n$, be members of $\Phi_{H}$ and consider the following system of $2 n^{2}$ nonlinear differential equations:

$$
\begin{aligned}
& d\left(y_{i j}\right) / d x=\sum_{h=1}^{n} k_{i h}\left[x, \phi_{i h}(Y), \sigma_{i h}(Z) ; \psi\right] \cdot z_{h j}, \\
& d\left(z_{i j}\right) / d x=\sum_{h=1}^{n} g_{i h}\left[x, \tau_{i h}(Y), \delta_{i h}(Z) ; \psi\right] \cdot y_{h j},
\end{aligned}
$$


on $X_{b}: 0 \leqq x \leqq b, L: d_{1}<\psi<d_{2}$, where the following conditions are imposed:

(i) The real-valued functions $k_{i j}[x, u, v ; \psi]$ and $g_{i j}[x, u, v ; \psi]$ are continuous in $(x, u, v, \psi)$ for all $x$ on $X_{b}$, for all $\psi$ on $L$, and for all real values of $u$ and $v$.

(ii) There exists a continuous function $m(x)$ such that for all $x$ on $X_{b}, \psi$ on $L$, and all real values of $u$ and $v$,

and

$$
\begin{aligned}
& \left|k_{i j}[x, u, v ; \psi]\right| \leqq m(x), \\
& \left|g_{i j}[x, u, v ; \psi]\right| \leqq m(x),
\end{aligned} \quad(i, j=1,2, \cdots, n) .
$$

(iii) The real-valued functions $k_{i j}[x, u, v ; \psi]$ and $g_{i j}[x, u, v ; \psi], i, j=1, \cdots, n$, satisfy a Lipschitz condition with respect to $u$ and $v$.

(iv) Each of $K[x ; Y ; Z ; \psi]$ and $-G[x ; Y ; Z ; \psi]$ is symmetric and positive definite for all $x$ on $X_{b}$, all $\psi$ on $L$, and all absolutely continuous matrices $Y$ and $Z$ such that for each $x$ on $X_{b}$ and $\psi$ on $L$

$$
\|Y(x, \psi)\|+\|Z(x, \psi)\| \leqq\{\|Y(0, \psi)\|+\|Z(0, \psi)\|\} \cdot \exp \left[\int_{0}^{x} n^{2} \cdot m(t) d t\right] .
$$

(v) For each $x$ on $X_{b}$, for all $\psi$ on $L$, and for all absolutely continuous matrices $Y$ and $Z$ satisfying the bound in (iv); either $K[x ; Y ; Z ; \psi]-(-G[x ; Y ; Z ; \psi])$ or $-G[x ; Y ; Z ; \psi]-K[x ; Y ; Z ; \psi]$ is nonnegative definite.

(vi) There exists a positive number $m(K, G)$ such that if $k_{i}[x ; Y ; Z ; \psi]$ and $g_{i}[x ; Y ; Z ; \psi], i=1,2, \cdots, n$, are the $i$ th characteristic roots of $K$ and $G$ respectively for each fixed $(x, \psi)$ on $X_{b} L$ and if $\alpha(x)=\max \left(k_{i},-g_{i}\right), 1 \leqq i \leqq n, x$ on $X_{b}$, for all $\psi$ on $L$ and $\beta(x)=\min \left(k_{i},-g_{i}\right), 1 \leqq i \leqq n, x$ on $X_{b}$, for all $\psi$ on $L$, then for each $x$ on $X_{b}, \alpha(x) / \beta(x) \leqq m(K, G)$.

As in $\S 2$, we shall represent system (3.3) in the matrix form

$$
\begin{aligned}
& d Y / d x=K[x ; Y ; Z ; \psi] \cdot Z, \\
& d Z / d x=G[x ; Y ; Z ; \psi] \cdot Y .
\end{aligned}
$$

THEOREM 3.2. If $K$ and $G$ satisfy conditions (i)-(vi) and $\{Y(x), Z(x)\}$ is the solution pair of (3.4) satisfying the initial condition

$$
Y(0, \psi) \equiv 0 \text { on } L, \quad Z(0, \psi)=A(\psi),
$$

$A(\psi)$ nonsingular on $L$, then there exists a continuous, symmetric matrix $Q(x, \psi)$ and a nonsingular, continuously differentiable matrix $R(x, \psi)$ such that

$$
\begin{aligned}
& Y(x, \psi)=S^{*}[x ; Q ; \psi] \cdot R(x, \psi), \\
& Z(x, \psi)=C^{*}[x ; Q ; \psi] \cdot R(x, \psi)
\end{aligned}
$$

on $X_{b}$ for each $\psi$ on L. Furthermore, $R(x, \psi)$ satisfies the differential system

$$
\begin{aligned}
& R^{\prime}(x, \psi) \\
& \quad=\left\{S[x ; Q ; \psi] \cdot K \cdot C^{*}[x ; Q ; \psi]+C[x ; Q ; \psi] \cdot G \cdot S^{*}[x ; Q ; \psi]\right\} R(x, \psi)
\end{aligned}
$$




$$
R(0, \psi)=A(\psi)
$$

on $X_{b}$ for each $\psi$ on $L$, and $Q(x, \psi)$ is the matrix

$$
Q(x, \psi)=C[x ; Q ; \psi] \cdot K \cdot C^{*}[x ; Q ; \psi]-S[x ; Q ; \psi] \cdot G \cdot S^{*}[x ; Q ; \psi] .
$$

Moreover, $Q(x, \psi)$ has the following properties on $X_{b} L$ :

(i) $Q(x, \psi)$ satisfies conditions (i), (ii), (iii) imposed on system (3.1), (3.2),

(ii) $\min [\operatorname{tr} K, \operatorname{tr}(-G)] \leqq \operatorname{tr} Q \leqq \max [\operatorname{tr} K, \operatorname{tr}(-G)]$.

Proof. For each $\psi$ on $L$, the first part of the theorem is merely a restatement of Theorem 2.1. Thus it remains to show that the matrix $Q(x, \psi)$ has properties (i) and (ii) above.

(i) $Q(x, \psi)$ is continuous, symmetric and positive definite on $X_{b} L$ since each of $K$ and $-G$ has these properties on $X_{b} L$ and each of $S(x, \psi)$ and $C(x, \psi)$ is continuous on $X_{b} L$. Fix any $(x, \psi)$ and assume that $K-(-G)$ is nonnegative definite at $(x, \psi)$. Let $\phi$ be the unit vector corresponding to the maximum characteristic root $q_{1}$ of $Q(x, \psi)$. Then

$$
\begin{aligned}
q_{1}=\phi Q \phi^{*} & =(\phi C) \cdot K \cdot(\phi C)^{*}+(\phi S) \cdot(-G) \cdot(\phi S)^{*} \\
& \leqq(\phi C) \cdot K \cdot(\phi C)^{*}+(\phi S) \cdot K \cdot(\phi S)^{*} \\
& \leqq \alpha \cdot\|\phi C\|^{2}+\alpha \cdot\|\phi S\|^{2}=\alpha,
\end{aligned}
$$

where $\alpha=\max \left(k_{i},-g_{i}\right)$ as defined in (vi) of system (3.3). Similarly, if $\delta$ is the unit vector corresponding to the minimum characteristic root $q_{n}$ of $Q(x, \psi)$, then

$$
\begin{aligned}
q_{n}=\delta Q \delta^{*} & =(\delta C) \cdot K \cdot(\delta C)^{*}+(\delta S) \cdot(-G) \cdot(\delta S)^{*} \\
& \geqq(\delta C) \cdot(-G) \cdot(\delta C)^{*}+(\delta S) \cdot(-G) \cdot(\delta S)^{*} \\
& \geqq \beta \cdot\|\delta C\|^{2}+\beta \cdot\|\delta S\|^{2}=\beta,
\end{aligned}
$$

where $\beta=\min \left(k_{i},-g_{i}\right)$ as defined in (vi).

Thus, for each $(x, \psi)$ on $X_{b} L$, the maximum characteristic root of $Q(x, \psi)$ is not greater than the maximum of the characteristic roots of the matrices $K$ and $-G$; and the minimum characteristic root of $Q(x, \psi)$ is not less than the minimum of the characteristic roots of the matrices $K$ and $-G$. It now follows that

$$
[\operatorname{tr} Q(x, \psi)] / \lambda(x) \leqq[n \cdot \alpha(x)] / \beta(x) \leqq n \cdot m(K, G),
$$

where $\lambda(x)$ is the function defined in (iii) of system (3.1), (3.2).

To establish property (ii), fix any $(x, \psi)$ on $X_{b} L$ and assume that $K-(-G)$ is nonnegative definite at $(x, \psi)$. By Lemma 2.3,

$$
\operatorname{tr} K=\operatorname{tr} C \cdot K \cdot C^{*}+\operatorname{tr} S \cdot K \cdot S^{*}
$$

and 


$$
\operatorname{tr}(-G)=\operatorname{tr} C \cdot(-G) \cdot C^{*}+\operatorname{tr} S \cdot(-G) \cdot S^{*} .
$$

Since we assumed that $K-(-G)$ is nonnegative definite, $\operatorname{tr} S \cdot K \cdot S^{*} \geqq$ $\operatorname{tr} S \cdot(-G) \cdot S^{*}$ and $\operatorname{tr} C \cdot(-G) \cdot C^{*} \leqq \operatorname{tr} C \cdot K \cdot C^{*}$. Property (ii) now follows immediately. Thus the theorem is established.

We shall now impose the following two conditions on the parameter $\psi$ in order to investigate the oscillatory behavior of solutions of systems of from (3.4).

Condition A. There exists a number $p, p>d_{1}$, on $L$ such that if $s$ is any fixed value of $\psi$ on $d_{1}<\psi \leqq p$ and $M$ denotes the upper bound of $[\operatorname{tr} K, \operatorname{tr}(-G)]$ on $X_{b}$ for this value of $\psi$, then $M<\pi / b$.

Condition B. There exists a subinterval $D: c \leqq x \leqq d$ of $X_{b}$ such that if $m(\psi)=\min [\operatorname{tr} K, \operatorname{tr}(-G)]$ on $D$ for each fixed $\psi, \operatorname{then} \lim _{\psi \rightarrow d_{2}} m(\psi)=+\infty$.

Observe that Condition A insures that for every $\psi$ on $d_{1}<\psi \leqq p, \int_{0}^{b} \operatorname{tr} Q(x, \psi) d x$ $<\pi$ and consequently, by Corollary 1.4.2, $S[x ; Q ; \psi]$ is nonsingular on $0<x \leqq b$ for fixed values of $\psi$ on $d_{1}<\psi \leqq p$. Therefore, by Theorem 3.2, $Y(x, \psi)$ is nonsingular on $0<x \leqq b$ for fixed values of $\psi$ on $d_{1}<\psi \leqq p$.

Condition $B$ insures that $\lim _{\psi \rightarrow d_{2}} \int_{0}^{b} \operatorname{tr} Q(x, \psi) d x=+\infty$ since

$$
\begin{aligned}
\int_{0}^{b} \operatorname{tr} Q \geqq \int_{0}^{b} \min [\operatorname{tr} K, \operatorname{tr}(-G)] & \geqq \int_{c}^{d} \min [\operatorname{tr} K, \operatorname{tr}(-G)] \\
& \geqq m(\psi) \cdot(d-c) .
\end{aligned}
$$

Definition. A characteristic number of system (3.4) is a value of $\psi$ for which the system has a nonidentically singular solution.

Theorem 3.3. If, in system (3.4), Conditions $\mathrm{A}$ and $\mathrm{B}$ are satisfied, and if $\{Y(x, \psi), Z(x, \psi)\}$ is a solution pair of (3.4) satisfying the initial condition

$$
Y(0, \psi) \equiv 0 \text { on } L, \quad|Y(b, \psi)|=0,
$$

then there exists an increasing sequence, $i_{1}, i_{2}, \cdots$, of positive integers and an infinite collection, $k_{1}, k_{2}, \cdots$, of sets of characteristic numbers for the system (3.4), (3.10) such that if $e_{j}$ is a number in $k_{j}$, then $Y\left(x, e_{j}\right)$ has exactly $i_{j}$ singularities on $0<x \leqq b$. Moreover, for each positive integer $j$ there exists a positive integer $m$ such that for every positive integer q greater than $m$, every number in $k_{q}$ is greater than every number in $k_{j}$.

LEMMA 3.2. If the pair $\{Y(x, \psi), Z(x, \psi)\}$ is a solution of system (3.4) and $Y(0, \psi) \equiv 0$ on $L$, then $|Y(x, \psi)| \neq 0$ and $|Z(x, \psi)| \not \equiv 0$ on $X_{b}$ only in case $|Z(0, \psi)| \neq 0$.

Proof. If $s$ is a value of $\psi$ on $L$ such that $|Z(0, s)| \neq 0$, then, by Theorem 2.1, $Y(x, s)=S^{*}[x ; Q ; s] \cdot R(x, s)$ and $Z(x, s)=C[x ; Q ; s] \cdot R(x, s)$ where $R(x, s)$ is nonsingular on $X_{b}$. Consequently, $Y$ and $Z$ are not identically singular on $X_{b}$. 
If $t$ is a value of $\psi$ on $L$ such that $|Z(0, t)|=0$, then by the remark following the proof of Theorem 2.1, it follows that $Y$ and $Z$ are identically singular on $X_{b}$.

From this lemma we can conclude that if $\psi$ is to be a characteristic number for the system (3.4), (3.10), then $|Z(0, \psi)| \neq 0$; and, in this case, Theorem 3.2 applies and we can write

$$
Y(x, \psi)=S^{*}[x ; Q ; \psi] \cdot R(x, \psi), \quad Z(x, \psi)=C^{*}[x ; Q ; \psi] \cdot R(x, \psi),
$$

where $R(x, \psi)$ is nonsingular on $X_{b} L$.

Proof of the theorem. As $\psi$ increases from a value less than $p$ to $d_{2}, \int_{0}^{b} \operatorname{tr} Q(x, \psi) d x$ increases from a value less than $\pi$ to $+\infty$. It follows from the continuity of $\int_{0}^{b} \operatorname{tr} Q(x, \psi) d x$ in $\psi$ and from Theorem 3.1 that the number of singularities of $S[x ; Q ; \psi]$ increases without bound. Hence let $k_{j}$ be the totality of values of $\psi$ on $L$ which have the property that $|S[b ; Q ; \psi]|=0$ and $S[x ; Q ; \psi]$ has exactly $i_{j}-1$ singularities on $0 \leqq x<b$. By Theorem 3.1 and the fact that $\lim _{\psi \rightarrow d_{2}} \int_{0}^{b} \operatorname{tr} Q(x, \psi) d x=+\infty$, there exists a number $t_{j}$ on $L$ such that $S[x ; Q ; \psi]$ has more than $i_{j}$ singularities on $X_{b}$ for every value of $\psi$ on $t_{j} \leqq \psi<d_{2}$. Thus no values of $k_{j}$ can lie on $t_{j} \leqq \psi<d_{2}$. It follows from Theorem 1.7 that there exists a positive integer $m$ such that $S[x ; Q ; \psi]$ has less than $m$ singularities on $X_{b}$ for all $\psi$ on $p \leqq \psi \leqq t_{j}$. Thus no values of $k_{m}, k_{m+1}, \cdots$ can lie on $p \leqq \psi \leqq t_{j}$. Therefore, for any $q$ greater than $m$, every number in $k_{q}$ is greater than every number in $k_{j}$. By Theorem 3.2, the singularities of $Y(x, \psi)$ coincide with the singularities of $S[x ; Q ; \psi]$ which completes the proof of the theorem.

A consideration of the two point boundary condition

$$
Y(0, \psi) \equiv 0 \text { on } L, \quad|Z(b, \psi)|=0
$$

yields the following theorem:

THEOREM 3.4. If, in system (3.4), Conditions $\mathrm{A}$ and $\mathrm{B}$ are satisfied, and if $\{Y(x, \psi), Z(x, \psi)\}$ is a solution pair of (3.4), (3.11), then there exists an increasing sequence, $j_{1}, j_{2}, \cdots$, of positive integers and an infinite collection, $h_{1}, h_{2}, \cdots$, of sets of characteristic numbers for the system such that if $e_{i}$ is any value in $h_{i}$, then $Z\left(x, e_{i}\right)$ has exactly $j_{i}$ singularities on $X_{b}$. Moreover, for each positive integer $i$ there exists a positive integer $m$ such that for every positive integer $q$ greater than $m$, every number in $h_{q}$ is greater than every number on $h_{i}$.

Proof. The proof proceeds in the same manner as Theorem 3.3.

Interchanging the roles of $Y(x, \psi)$ and $Z(x, \psi)$ in Theorem 3.2 and repeating the foregoing discussion, we obtain results analogous to Theorem 3.3 and 3.4 for the system (3.4) together with the boundary conditions of the form:

$$
\begin{array}{ll}
Z(0, \psi) \equiv 0 \text { on } L, & |Z(b, \psi)|=0, \\
Z(0, \psi) \equiv 0 \text { on } L, & |Y(b, \psi)|=0 .
\end{array}
$$




\section{BIBLIOGRAPHY}

1. J. H. Barrett, Matrix systems of second order differential equations, Portugal. Math. 14 (1955), 78-89.

2. - A Prüfer transformation for matrix differential equations, Proc. Amer. Math. Soc. 8 (1957), 510-517.

3. E. A. Coddington and N. Levinson, Theory of ordinary differential equations, McGrawHill, New York, 1955.

4. E. O. Ince, Ordinary differential equations, Dover, New York, 1926.

5. F. J. Murray and K. S. Miller, Existence theorems for ordinary differential equations, New York Univ. Press, New York, 1954.

6. W. T. Reid, A matrix differential equation of the Riccati type, Amer. J. Math. 68 (1964), 237-246.

7. - A Prüfer transformation for differential systems, Pacific J. Math. 8(1958), 575-584.

8. W. M. Whyburn, Existence and oscillation theorems for non-linear differential equations of second order, Trans. Amer. Math. Soc. 30 (1928), 848-854.

9. - On the fundamental existence theorems for differential systems, Ann. of Math. 30 (1928), 31-38.

10. - Functional properties of the solutions of differential systems, Trans. Amer. Math. Soc. 32 (1930), 502-508.

Headquarters, National Aeronautics and Space. Administration, WASHINGTON, D.C.

UNIVERSITY OF NORTH Carolina,

Chapel Hil.t., North Carolina 